\section{Application of two in vitro methods for the toxicity test of autogenous vaccines}

Erika Molica Colella,1 Silvia Dotti,1 Riccardo Villa, 1 Guerino Lombardi, 2 Massimo Amadori ${ }^{3}$

1Centro di Referenza Nazionale per i Metodi Alternativi, Benessere e Cura degli Animali da Laboratorio, Istituto Zooprofilattico Sperimentale della Lombardia e dell'Emilia Romagna (IZSLER), Brescia; ${ }^{2}$ Centro di Referenza Nazionale per la Formazione in Sanità Pubblica Veterinaria, Istituto Zooprofilattico Sperimentale della Lombardia e dell'Emilia Romagna (IZSLER), Brescia; ${ }^{3}$ Laboratorio Benessere Animale, Biochimica Clinica e Immunologia Veterinaria, Istituto Zooprofilattico Sperimentale della Lombardia e dell'Emilia Romagna (IZSLER), Brescia, Italy

\begin{abstract}
According to the 3Rs principle (Replacement, Refinement, Reduction), this study aims to find alternative methods to evaluate the toxicity of autogenous vaccines. Currently in Italy the Istituti Zooprofilattici Sperimentali (II.ZZ.SS.) must perform the in vivo toxicity test for each lot of autogenous vaccine produced as laid down in the Decree of 17 March 1994. This paper describes two in vitro methods for assessing the toxicity of autogenous vaccines. The first is the MTT test based on the metabolic reaction of tetrazolium salt in vital cells. The second method is the test for measurement of IL-1ß production by macrophages, obtained after in vitro differentiation from pig monocytes in peripheral blood mononuclear cells. The two tests were performed on different vaccine antigens dilution: 1:20, 1:100 and 1:500. The results show a positive tendency between the two methods pointing out the potential of these methodologies combined for the replacement of the current in vivo test.
\end{abstract}

\section{Introduction}

Autogenous vaccines are immunizing products prepared against pathogenic microorganisms isolated from sick animals of one herd and only usable in the same farm. The Decree of 17 March 1994 in Italy regulates the production and control of autogenous veterinary vaccine. The legislation provides that the II.ZZ.SS. are the only agencies authorized by the Ministry of Health for the production of veterinary autogenous and autologous vaccines. Moreover, as required by the law, the II.ZZ.SS. must perform the in vivo toxicity test (mouse model) for each lot of vaccine produced. ${ }^{1}$ According to the 3 Rs principle, this study aims to find alternative methods to evaluate the vaccines toxicity replacing the animal model.

\section{Materials and Methods}

In this study, the MTT in vitro method was applied to evaluate the cytotoxicity of autogenous vaccine antigens. Moreover, an in vitro method was developed to measure the release of interleukin IL-1 $\beta$ by porcine macrophages in order to evaluate the potential in vitro inflammatory response triggered by the vaccine. The antigens analysed in the tests were inactivated with $0.8 \%$ formalin for 24 hours at $37^{\circ} \mathrm{C}$ and washed with saline solution, before the addition of the adjuvant.

The first method used is the cytotoxicity test MTT, based on the metabolic reaction of tetrazolium salt in vital cells. The MTT test is one of the most widely used, in accordance with the UNI EN ISO 10993-5 standard, concerning the biological evaluation of medical devices, which allows evaluating the safety and toxic potential of products. ${ }^{2}$ For the test, the L-929 cell line was used, seeded at the concentration of $1.0 \times 10^{5}$ cells $/ \mathrm{mL}$ in 96-well plates in MEM culture medium added with $10 \%$ of foetal bovine serum (FBS), and incubated at $+37^{\circ} \mathrm{C} \pm$ $1{ }^{\circ} \mathrm{C}, 5 \%$ of $\mathrm{CO}_{2}$. After an overnight incubation, the vaccine antigens were diluted and distributed $100 \mu \mathrm{L} /$ well. After 24 hours of incubation, the cell monolayer was treated with MTT salt and the intensity of the colorimetric reaction was correlated to cell viability.

The second method performed is a test for the assessment of IL-1ß production by porcine macrophages in response to vaccine antigens. Interleukin-1ß (IL-1ß) is a proinflammatory cytokine mainly produced by activated macrophages and monocytes. The precursor (pro IL-1ß) is located at cytoplasmic level and must be cleaved by caspase- 1 to generate the mature activated form. ${ }^{3}$ Assessment of IL-1ß production by macrophages in response to vaccine anti-
Correspondence: Erika Molica Colella, Centro di Referenza Nazionale per i Metodi Alternativi, Benessere e Cura degli Animali da Laboratorio, Istituto Zooprofilattico Sperimentale della Lombardia e dell'Emilia Romagna (IZSLER), Brescia, Italy.

E-mail: erika.molica@gmail.com

Key words: Autogenous vaccines; toxicity tests; in vitro methods; MTT, IL-1 $\beta$.

Conference presentation: this paper was presented at the Second Centro 3R Annual Meeting - 3Rs in Italian Universities, 2019, June 20-21, University of Genoa, Italy.

Received for publication: 28 October 2019.

Accepted for publication: 11 November 2019.

This work is licensed under a Creative Commons Attribution NonCommercial 4.0 License (CC BY-NC 4.0).

CC Copyright: the Author(s), 2019

Licensee PAGEPress, Italy

Biomedical Science and Engineering 2019; 3(s3):114 doi:10.4081/bse.2019.114

gens could help evaluate the safety and efficacy of the vaccine-induced immune response. In particular, macrophages were

obtained after in vitro differentiation from peripheral blood mononuclear cells (PBMC), using $10 \mathrm{ng} / \mathrm{mL}$ of MacrophageColony Stimulating Factor (M-CSF). Differentiated macrophages were reacted with the same antigens at different dilutions for 24 hours, followed by quantification of released IL-1ß by "Duo set ELISA for Porcine IL-1ß/IL-1F2” (R\&D System).

\section{Results}

The results showed two crucial points to consider for the IL-1ß assessment in vitro test: i) the choice of the cell population, because macrophages with high basal reactivity tended to provide a plateau response; ii) the need to dilute the antigens because their high bacterial content caused an effect "endotoxin tolerance-like", that inhibits the release of IL-1ß as showed in Figure 1.4

Furthermore, the MTT test results showed that the samples are cytotoxic at lower dilutions, showing a cell viability $\geq 70 \%$ from at least $1: 8$. The maximum dilution, to which a cytotoxic effect was so far detected, looks to be 1:128.

Moreover, to assess the correlation between the two methods, the results of the MTT test at the dilutions 1:16 were trans- 


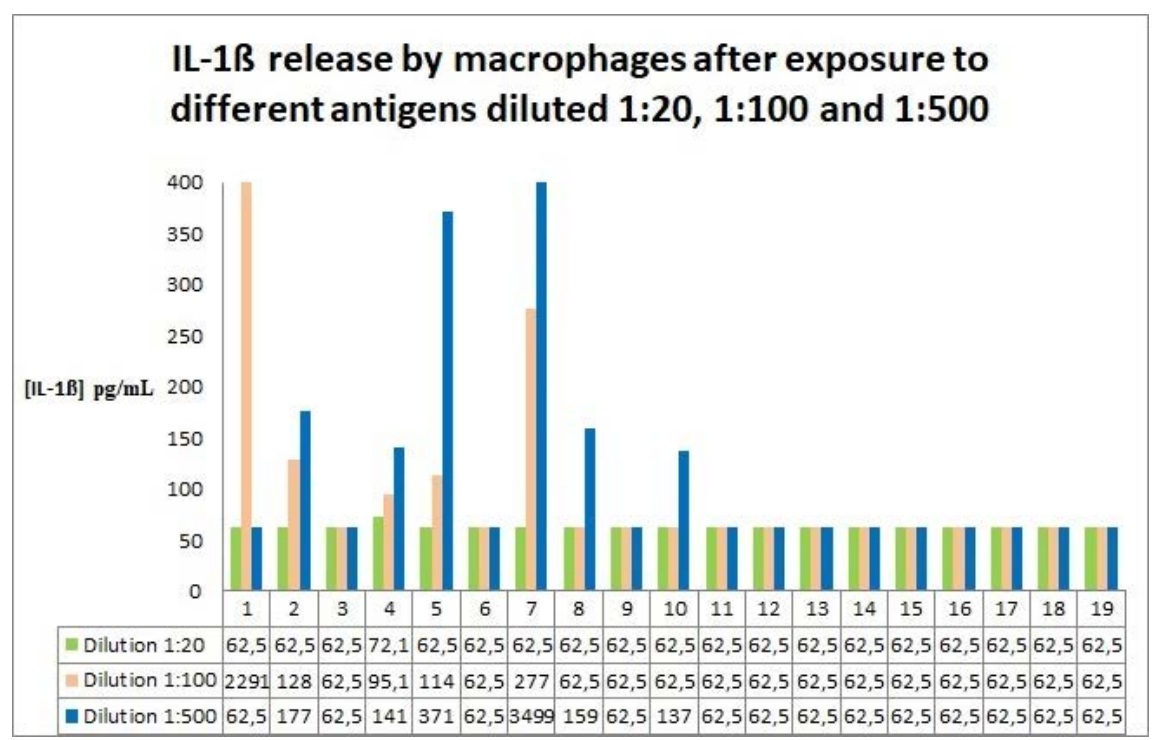

Figure 1. IL-1 $\beta$ release by macrophages after exposure to different antigens.

formed into a percentile value and were correlated via GraphPad Prism software with the concentrations of IL- $1 \beta$ detected at 1:500 dilutions of the antigens.

The statistical analysis, carried out

\section{Conclusions} using the Spearman coefficient, shows a positive tendency between the two methods $(\mathrm{R}=0.4955, \mathrm{P}=0.03)$ pointing out the poten- tial of these combined methodologies to examine different features of the immune/toxic response, that autogenous vaccine could trigger in the target animals. ${ }^{5}$

The study confirms the scientific value of the 3Rs and their applicative potentiality, especially when alternative in vitro methods are combined in integrated assay strategies to enhance, through multiple end-points, the effectiveness and predictive capacity of the models.

\section{References}

1. Ministerial Decree 17 March 1994.

2. UNI EN ISO 10993-5: 2009. Biological evaluation of medical devices Tests for in vitro cytotoxicity. 2009.

3. Martinon F, Burns K, Tschopp J. The inflammasome: a molecular platform triggering activation of inflammatory caspases and processing of pro-IL-1ß. Mol Cell 2002;10:417-26.

4. West MA, Heagy W. Endotoxin tolerance: a review. Crit Care Med 2002;30: S64-73.

5. Spearman C. The proof and measurement of association between two things. Am J Psychol 1904;15:72-101. 